\title{
Short hairpin RNA targeting FOXQ1 inhibits invasion and metastasis via the reversal of epithelial- mesenchymal transition in bladder cancer
}

\author{
ZHAOHUI ZHU* ${ }^{*}$ ZHINENG ZHU* , ZILI PANG, YIFEI XING, \\ FENG WAN, DONGYANG LAN and HAIPENG WANG \\ Department of Urology, Union Hospital, Tongji Medical College, Huazhong University of \\ Science and Technology, Wuhan 430022, Hubei, P.R. China
}

Received November 6, 2012; Accepted December 21, 2012

DOI: $10.3892 /$ ijo.2013.1807

\begin{abstract}
The epithelial-mesenchymal transition (EMT) promotes cancer invasion and metastasis, however, the integrative mechanisms that coordinate the process are incompletely understood. In this study, we defined a pivotal functional role for the Forkhead transcription factor FOXQ1 in regulating EMT in bladder cancer. We initially investigated the expression of FOXQ1, TGF- $\beta 1$ and EMT biomarkers E-cadherin, Vimentin in 65 cases of bladder transitional cell carcinoma (BTCC) specimens by reverse transcription-polymerase chain reaction (RT-PCR), western blot analysis and immunohistochemistry. Search results indicated that FOXQ1 expression was inversely correlated to E-cadherin, but positively to TGF- $\beta 1$ and Vimentin in patients with BTCC $(\mathrm{P}<0.05)$. Furthermore, we aimed to construct short hairpin RNA (shRNA) expression plasmids against the FOXQ1 gene and transfect shRNAs into high metastatic potential human bladder cancer T24 cells with Lipofectamine 2000. RNAimediated suppression of FOXQ1 expression reversed the EMT process accompanied by upregulation of E-cadherin, as well as a loss expression of Vimentin in highly invasive T24 cells $(\mathrm{P}<0.05)$. The inhibition of FOXQ1 expression with shRNA vector also led T24 cells to acquire an epithelial cobblestone phenotype, significantly reduced motility and subsequent invasiveness of bladder cancer cells $(\mathrm{P}<0.05)$. In conclusion that FOXQ1 may be a novel EMT-inducing transcription factor through controlling the expression of E-cadherin and aggressiveness of cancer cells and targeting the transcription factor FOXQ1 could hence serve as a novel therapeutic strategy for cancer patients.
\end{abstract}

Correspondence to: Dr Zili Pang and Dr Yifei Xing, Department of Urology, Union Hospital, Tongji Medical College, Huazhong University of Science and Technology, 1277 Jiefang Avenue, Wuhan 430022, Hubei, P.R. China

E-mail: zilipang027@gmail.com

E-mail: yfxing@yahoo.com

*Contributed equally

Key words: transcription factor FOXQ1, epithelial-mesenchymal transition, bladder cancer, short hairpin RNA

\section{Introduction}

Metastasis of tumor cells to distant organs is the most common cause of death arising from human carcinomas. During the metastatic cascade, carcinoma cells often initiate a transdifferentiation step known as epithelial-mesenchymal transition (EMT), one dynamic cellular process deemed to underlie metastasis by promoting acquisition of migratory and invasive abilities $(1,2)$. EMT is characterized by loss of E-cadherin that is proposed to be a critical switch of EMT, accompanied with increased expression of mesenchymal cell phenotype markers (Vimentin, $\mathrm{N}$-cadherin and fibronectin) (3), which contribute to disruption of cell adherens junction and the ensuing loss of cell polarity. Subsequently, epithelial cells acquire a fibroblastic phenotype, dissociate from the epithelium and migrate to distant organs. Therefore, EMT underlying metastasis remains an important aspect of cancer research.

During the acquisition of EMT phenotype, several transcription factors including the Snail-family members Snail1, Snail2 (formerly Slug), Twist1, E47 and the Zeb-family members Zeb1 and Zeb2 (Sip1) are known to potently modulate epithelial cell plasticity and induce EMT phenotype by repressing E-cadherin expression (4-7). The transcription factor overexpression in human carcinomas binds to E-box elements within the promoter region of E-cadherin, leading to transcription inhibition of E-cadherin and triggering EMT in cancer cells, which suggest that transcription factors play a key role in controlling the induction of EMT phenotype.

Members of the Forkhead box protein family of transcription factors (Fox-factors) have been shown to be involved in regulating EMT of epithelial cancer cells (8). In humans, this transcription factor family characterized by the presence of a DNA-binding domain called the Forkhead box or winged helix domain comprises 17 subfamilies (FoxA-FoxR) with 43 known members $(9,10)$. FOXC2 and FOXM1 have been recently implicated in aggressive basal-like breast cancer with a role in regulating EMT and metastasis $(11,12)$. FOXQ1 belongs to the human Forkhead box gene family (13). It has been recognized that FOXQ1 as a potent modulator of epithelial cell plasticity and is involved in epithelial 
differentiation and cell proliferation which regulates the developmental function of many organs in the body (14). Moreover, overexpression of FOXQ1 has been reported in several cancers including lung carcinoma cell lines, pancreatic ductal adenocarcinomas (15) and in the transition from normal intestinal epithelium to adenoma and carcinomas in an APC min/t mouse (16). Interestingly, higher expression of FOXQ1 in human colorectal carcinomas has been reported to directly control the expression of $\mathrm{p} 21^{\text {Wafl/Cip1 }}$ and enhance tumorigenicity and tumor progression and found to be associated with poor prognosis of colorectal cancer patients (17). Furthermore, emerging evidence suggests that FOXQ1 was identified as transcriptionally induced in a TGF- $\beta 1$ responsive cell culture model of cytokine-induced EMT-like progression (18), suggesting a potential impact of FOXQ1 expression in the regulation of EMT. FOXQ1 has been confirmed to be increased in expression in a Smad4-dependent manner in response to TGF- $\beta 1$ treatment (19), demonstrating that the expression of FOXQ1 is regulated by TGF- $\beta 1$ which is profoundly known as a major inducer of EMT processes and plays an important role in the outcome of TGF- $\beta 1$ signaling. These results strongly suggest that FOXQ1 may have a crucial role in the development and progression of human cancers. However, the precise mechanism for FOXQ1 inducing EMT phenotype and tumor progression in the transitional cell carcinoma (TCC) has not been elucidated. No previous study has examined the effect of targeted inhibition or downregulation of FOXQ1 in bladder cancer cells.

In this research, to study the functional significance of EMT in initiating metastasis of bladder cancer, we used short hairpin RNA (shRNA) against FOXQ1 gene to implement gene silencing by means of RNA interference in the human bladder cancer cell line T24 which has high metastatic potential. Then investigated the regulatory effect of FOXQ1 on gene expression of EMT molecular markers and further inspected its role in cell proliferation, cell migration and invasion with in vitro transfection assays. We showed that silencing of the FOXQ1 gene induced changes in markers of epithelial and mesenchymal phenotypes and the morphology of cells, displaying a mesenchymal-epithelial transition (MET). We further demonstrated that the inhibition of the FOXQ1 expression significantly depressed motility, subsequent migration and invasion of bladder cancer cells.

\section{Materials and methods}

Cell line culture and maintenance. The human bladder cancer cell line T24 was chosen for knockdown expression of FOXQ1 by stable transfection of FOXQ1 shRNA especially because T2 4 cells showed overexpression of FOXQ1 compared with BIU-87 cells. T24 cells are a cell line derived from a grade III bladder carcinoma, which are poorly differentiated and possess a higher potential of metastasis (20). T24 cells were maintained in our laboratory. They were cultured in RPMI1640 (Gibco, Rockville, MD) with 10\% fetal bovine serum (FBS) (Hyclone, Logan, OR) at $37^{\circ} \mathrm{C}$ under $5 \% \mathrm{CO}_{2}$ and $95 \%$ humidified air.

Tissue samples. This study was approved by the institutional Health Care/Hospital Ethics Committees. Informed consent was obtained from all patients prior to the study. Bladder tissue samples were obtained from 65 patients (mean age 65.2 years, range 45-78) with primary histologically-proven urothelial bladder cancer. For each specimen, the cancer tissue, paracarcinoma tissue $>1.5-2 \mathrm{~cm}$ from cancer tissue and its remote normal mucosa were analyzed and compared. These patients underwent transurethral resection (TUR-Bt), partial cystectomy, or radical cystectomy between 2009 and 2011 at the Department of Urology, Union Hospital of Tongji Medical College, Huazhong University of Science and Technology. None of the patients had received preoperative treatment. The samples collected displayed papillary or sessile appearance during the endoscopic procedure. The tumors were stratified as non-muscle invasive bladder cancer (NMIBC) or muscle invasive bladder cancer (MIBC) according to the 2002 UICC TNM classification for the stage (21), as shown in their respective cystectomy specimens. Grade was assigned using 2004 WHO/ISUP classification (22) (LMP, low malignant potential; LG, low grade; HG, high grade) which showed LMP in 18 cases, LG in 27 cases and HG in 20 cases. Tissue specimens from surgically resected bladder tumors were confirmed by pathology and immunostaining was evaluated by 2 independent pathologists to validate the diagnosis. The corresponding clinical information was obtained from the Union Hospital of Tongji Medical College.

Transient transfection. The short hairpin RNA eukaryotic expression vector targeting FOXQ1 (referring to GenBank NM_033260.3) proceeded by method of chemical synthesis according to the principle of design purchased from GenePharma Co., Ltd. (pGPU6/GFP/Neo; Shanghai, China). The targeting sequences of three different shRNAs and one negative control shRNA were designed using RNAi algorithm available online, one sequence with the most effective silencing effect targeting FOXQ1 was chosen for knockdown expression of FOXQ1 and subsequent experiment. The RNAi vector can be distinguished by neomycin resistance screening marker and green fluorescent protein (GFP) expression driven by the U6 promoter. T24 cells were seeded at a density of $6 \times 10^{5}$ cells per 24 -well plate in RPMI1640 with $10 \%$ FBS and grown overnight. Then preserved with RPMI-1640 free of serum or antibiotics and prepared for transfection assays. The cells were divided into two groups: shRNA-FOXQ1 and shRNA-NC (non-specificity sequence for a negative control). When the cells were 90-95\% confluent after washing twice with $1 \mathrm{ml}$ phosphate buffer saline (PBS), the shRNA plasmid was transfected into T24 cells using Lipofectamine 2000 transfection reagent (Invitrogen, Carlsbad, CA) at the ratio of 1:2. After $24 \mathrm{~h}$, GFP activity of the cells was detected by IX71 fluorescent microscope (Olympus, Tokyo, Japan).

$R T-P C R$. To measure the relative expression of mRNAs of each sample, total RNA was isolated from the indicated tissues, transfected and control bladder cancer cells, using TRIzol reagent (Invitrogen) according to the manufacturer's protocol. After being washed with 75\% ethanol, the final RNA extracts were eluted in a $20 \mu \mathrm{l}$ volume of distilled water treated with diethyl pyrocarbonate. The concentration and purity of RNA were measured with a spectrophotometer. All the RNA 
preparations had an optical density OD 260:OD 280 ratio of 1.8-2.0. Then, total RNA from each sample was reverse transcripted (RT) in a first-strand cDNA synthesis reaction with PrimeScript RT-PCR kit as recommended by the supplier (Takara Biotechnology Dalian, China), by reverse transcription at $37^{\circ} \mathrm{C}$ for $25 \mathrm{~min}$, followed by incubation at $85^{\circ} \mathrm{C}$ for $5 \mathrm{sec}$ in $20 \mu \mathrm{l}$ of reaction volume. Resultant cDNA $(1 \mu \mathrm{g})$ was used for semiquantitative polymerase chain reaction (PCR) amplification, which was performed with primers (Invitrogen) for: FOXQ1 5'-ATTTCTTGCTATTGACCGATGC-3' (sense) and 5'-CCCAAGGAGACCACAGTTAGAG-3' (antisense), TGF- $\beta 1$ 5'-AACCCACAACGAAATCTA-3' (sense) and 5'-TGAGGTAT CGCCAGGAAT-3' (antisense), E-cadherin 5'-AACGCATTGC CACATACAC-3' (sense) and 5'-GAGCACCTTCCATGACA GAC-3' (antisense), Vimentin 5'-ACAGGCTTTAGCGAGT TATT-3' (sense) and 5'-GGGCTCCTAGCGGTTTAG-3' (antisense), GAPDH 5'-GGTGAAGGTCGGAGTCAACGG-3' (sense) and 5'-CCTGGAAGATGGTGATGGGATT-3' (antisense) served as an internal control to determine the relative amount of RNA of the samples in the process of RT-PCR. The PCR (32 cycles) was conducted in a Mastercycler thermal cycler (Eppendorf, Hamburg, Germany). Each cycle included denaturation $\left(94^{\circ} \mathrm{C}, 30 \mathrm{sec}\right)$, annealing $\left(58^{\circ} \mathrm{C}, 30 \mathrm{sec}\right)$ and extension $\left(72^{\circ} \mathrm{C}, 30 \mathrm{sec}\right)$ performed in $25 \mu \mathrm{l}$ of reaction mixture. The initial denaturation period was $4 \mathrm{~min}$ and the final extension was $7 \mathrm{~min}$. Amplified products were analyzed by DNA gel electrophoresis in $2.0 \%$ agarose and were visualized by ethidium bromide staining under ultraviolet illumination. The result of gel electrophoresis was analyzed by Quantity One 4.5.0 software for the optical density.

qRT-PCR. Quantification of mRNA expression was performed with $1 \mu \mathrm{l}$ of cDNA in a final volume of $20 \mu \mathrm{l}$ containing $10 \mu \mathrm{l}$ of SYBR Green master mixture from Takara Clontech (Kyoto, Japan) and 10 pmol of sense and antisense primers of FOXQ1 5'-ATTTCTTGCTATTGACCGATGC-3' (sense) and 5'-CCCA AGGAGACCACAGTTAGAG-3' (antisense), E-cadherin 5'-CTG GACGCTCGGCCTGAAGT-3' (sense) and 5'-GGGTCAGTAT CAGCCGCTTT-3' (antisense), Vimentin 5'-ACAGGCTTTAG CGAGTTATT-3' (sense)and5'-GGGCTCCTAGCGGTTTAG-3' (antisense) GAPDH 5'-GGTGAAGGTCGGAGTCAACGG-3' (sense) and 5'-CCTGGAAGATGGTGATGGGATT-3' (antisense) respectively. qPCR was carried out in a DNA engine, Option TM2 Real-Time Detector (Bio-Rad, Hercules, CA) using the following thermal cycling profile: $95^{\circ} \mathrm{C}$ for $30 \mathrm{sec}$, followed by 40 cycles of amplification $\left(95^{\circ} \mathrm{C}\right.$ for $5 \mathrm{sec}, 58^{\circ} \mathrm{C}$ for $30 \mathrm{sec}$ ). All samples were run in triplicate, the relative levels of individual mRNA in each sample transcript to control GAPDH were calculated using the $2^{-\Delta \Delta C t}$ method.

Western blotting. Similarly to shRNA transfected bladder cancer cells, T24 and bladder tissue samples and protein extracts were prepared by washing cells with PBS and lysing in RIPA buffer containing protease inhibitor. The concentrations of protein were detected by BCA protein assay. Proteins of the samples in each group were resolved on $10 \%$ sodium dodecyl sulfate polyacrylamide gel electrophoresis (SDS-PAGE) (Bio-Rad) and transferred to a NC membrane (Bio-Rad), blocked with TBST buffer composed of $50 \mathrm{mM}$ Tris (pH 7.6), $150 \mathrm{mM} \mathrm{NaCl}$ and $0.05 \%$ Tween supplemented with $5 \%$ fat-free milk at $4^{\circ} \mathrm{C}$ for $1 \mathrm{~h}$. The membrane was incubated overnight at $4^{\circ} \mathrm{C}$ in TBST containing 5\% bovine serum albumin (BSA) with rabbit anti-human monoclonal antibody FOXQ1 (Abcam) (1:500), TGF- $\beta 1$, E-cadherin and Vimentin (Cell Signaling Technology, Beverly, MA) (1:500). Rabbit anti-human GAPDH polyclonal antibody (Santa Cruz Biotechnology Inc., Santa Cruz, CA) (1:500) was used for detecting the internal control protein. Then, blots were washed 3 times using TBST for $1 \mathrm{~h}$ at room temperature. Antibody binding was detected using peroxidase-conjugated goat anti-rabbit IgG and visualized with ECL chemiluminescent reagents (Pierce Biotechnology, Rockford, IL) according to the manufacturer's instructions.

Immunohistochemical staining analysis. Immunohistochemical staining was done on conventional paraffin-embedded tissue sections obtained from normal bladder and different stages of bladder cancers. Tissue sections were incubated with monoclonal antibody at 1:60 dilution, followed by treatment with a streptavidin-peroxidase immunohistochemical staining kit (Zymed Laboratories Inc., South San Francisco, $\mathrm{CA})$, according to the manufacturer's protocol. Finally, tissue sections were counterstained with hematoxylin to discriminate nucleus from cytoplasm, upgraded alcohols, mounted and analyzed by standard light microscopy. Immunohistochemical staining of FOXQ1, TGF- $\beta 1$, Vimentin and E-cadherin was defined as detectable immunoreactions in perinuclear and/ or cytoplasm. Expression of FOXQ1, TGF- $\beta 1$ and Vimentin was considered negative when no or $<49 \%$ of the tumor cells were stained (23). Cancer cells that were immunostained with $<10 \%$ staining were defined as having a reduced E-cadherin expression (24).

Cell wound healing assay. Wound healing assay was performed to examine the capacity of cell migration and invasion. Briefly, T24 cells were transfected in a 24 -well plate with Lipofectamine 2000 after the cells grew to $90-95 \%$ confluence. At $48 \mathrm{~h}$ post-transfection, the wound was generated by scratching the surface of the plates with a $0-100-\mu 1$ pipette tip. Cell migration into the wounded empty space was photographed using a microscope after $24 \mathrm{~h}$, well images were evaluated and the relative migration distance was analyzed.

Matrigel invasion assay. Forty-eight hours after shRNA plasmid transfection, cells were harvested, counted and resuspended in serum-free RPMI-1640 culture medium. A total $200 \mu \mathrm{l}$ of 5,000 cells of T24 cells were added to the upper compartment of the matrigel invasion chamber (BD Biosciences, Bedford, MA) in 24-well tissue culture plates and $500 \mu 1$ of RPMI-1640 containing 10\% FBS was added to the lower compartment. After a 24 -h incubation period at $37^{\circ} \mathrm{C}$ in $5 \% \mathrm{CO}_{2}$, membranes were fixed in methanol and stained with crystal violet. Cells on the upper surface of the filter were removed carefully with a cotton swab and experiments were performed in triplicate. Invasion power of the cells was determined by counting the number of cells that have migrated to the lower side of the membrane under a light microscope. Five visual fields (x400) were determined in each chamber. Finally, the mean value of fields was calculated. 
A
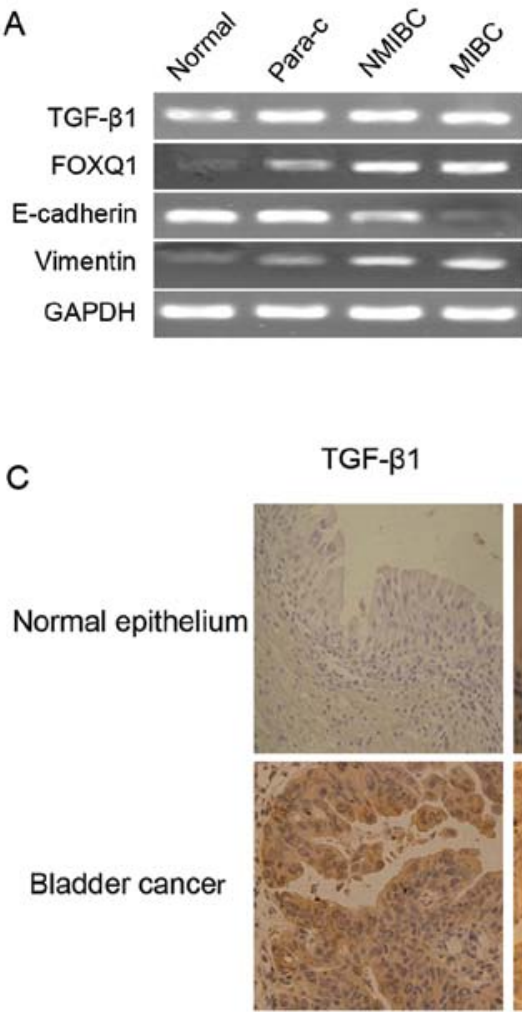

FOXQ1



B

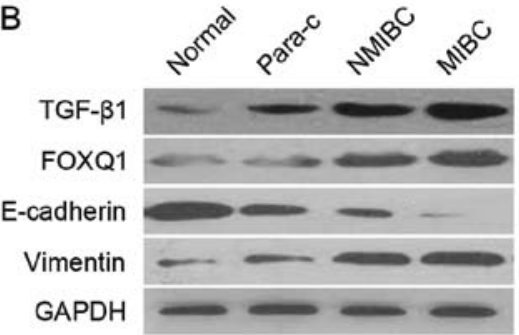

E-cadherin
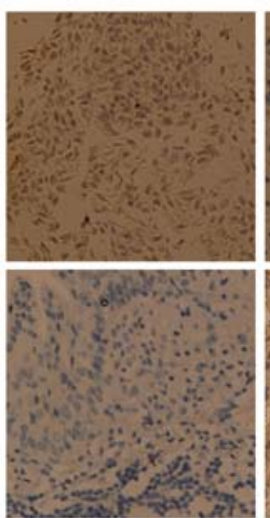

Vimentin

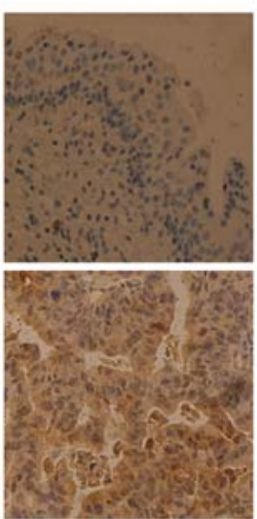

Figure 1. Expression levels of TGF- $\beta 1$, FOXQ1, E-cadherin and Vimentin in the normal bladder and tumor tissues. (A) RT-PCR (left panel) was performed using total RNA isolated from various samples derived from normal bladder tissues (normal), bladder paracancer tissues (para-c), non-muscle-invasive bladder cancer (NMIBC), muscle-invasive bladder cancer (MIBC), The experiment was repeated 3 times with reproducible results. (B) Protein levels of TGF- $\beta 1$, FOXQ1, E-cadherin and Vimentin in the bladder tissue samples, bladder cancer tissues were examined by western blot analysis (right panel) using corresponding monoclonal antibodies. (C) IHC staining of the normal bladder tissues, tumor tissues resected from bladder cancer patients with anti-TGF- $\beta 1$, anti-FOXQ1, anti-E-cadherin and anti-Vimentin antibodies was performed as described in Materials and methods. Original magnification, x200.

MTT assay. Bladder cancer cells described above were seeded at $4 \times 10^{3}$ cells per well in flat-bottomed 96-well plates. After $24 \mathrm{~h}$, cells were transduced with shRNA plasmid for 1,2, 3 and 4 days. At the end of culture, $10 \mu 1$ of MTT $(5 \mathrm{mg} / \mathrm{ml})$ was added to each well and plates were placed at $37^{\circ} \mathrm{C}$ for $4 \mathrm{~h}$. The medium was then removed and $100 \mu 1$ of dimethylsulfoxide (DMSO) solution was added to each well to lyse the cells. Absorbance was measured at 570 using a microplate reader to determine cell viability. Four replicate wells were tested per assay and each experiment was repeated 3 times.

Apoptosis assay. The effect of FOXQ1 silencing on cell apoptosis was determined by flow cytometry. Briefly, T24 cells at $1 \times 10^{6}$ cells per well were cultured in 6 -well plates overnight and transfected with $50 \mathrm{nM}$ of negative control shRNA-NC and FOXQ1-shRNA respectively. The cells were harvested and stained with Annexin-V and PI, using the Annexin V-FITC apoptosis detection kit (Keygentec, China). The apoptotic cells were assessed using a FACSCalibur instrument.

Statistical analysis. Data were expressed as the means \pm standard deviation and were analyzed using independent samples t-test by the statistical software program SPSS 17.0 (SPSS Inc., Chicago, IL). Values of $\mathrm{P}<0.05$ were considered statistically significant.

\section{Results}

High expression levels of TGF- $\beta 1, F O X Q 1$, Vimentin, or low level of E-cadherin in bladder cancer tissues. RT-PCR and western blot analysis were performed to determine whether a high-level of expression of FOXQ1 was accompanied by an alteration in the expression of epithelial markers, E-cadherin and Vimentin. Although there were significantly higher levels of FOXQ1, TGF- $\beta 1$ and Vimentin were also found in bladder cancer tissues of differential stages, paracancer tissues, in which E-cadherin expression levels were markedly decreased (Fig. 1A and B). FOXQ1 expression was inversely correlated to E-cadherin, but positively to Vimentin. Similar results could be obtained by IHC staining for detecting TGF- $\beta 1$, FOXQ1, Vimentin and E-cadherin in clinical tissue samples (Fig. 1C). The results from IHC staining indicated that FOXQ1 localized mainly in the cytoplasm in bladder cancer cells (Fig. 1C).

Higher expression of FOXQ1 in T24 cells compared with BIU-87 cells and the knockdown effect of FOXQ1-shRNA in T24 cells. To investigate the expression of FOXQ1, we analyzed the mRNA expression levels of FOXQ1 in human bladder cancer cell lines T24 and BIU-87 using qRT-PCR. High levels of FOXQ1 expression were observed in the highly invasive and mesenchymal-like bladder cancer T24 cells, relatively weak expression levels were detected in the 
A
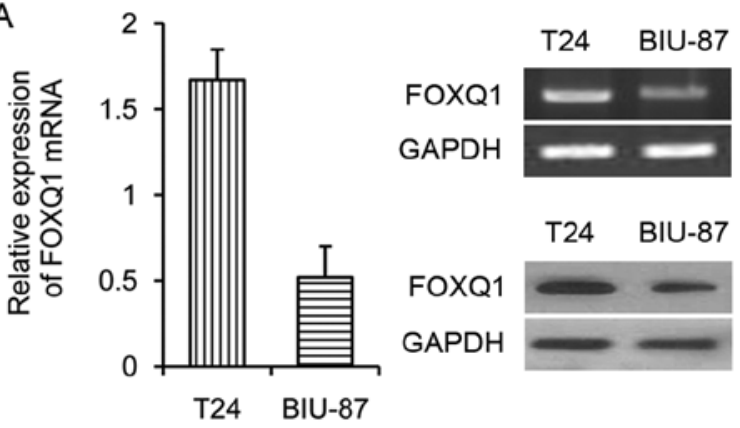

B

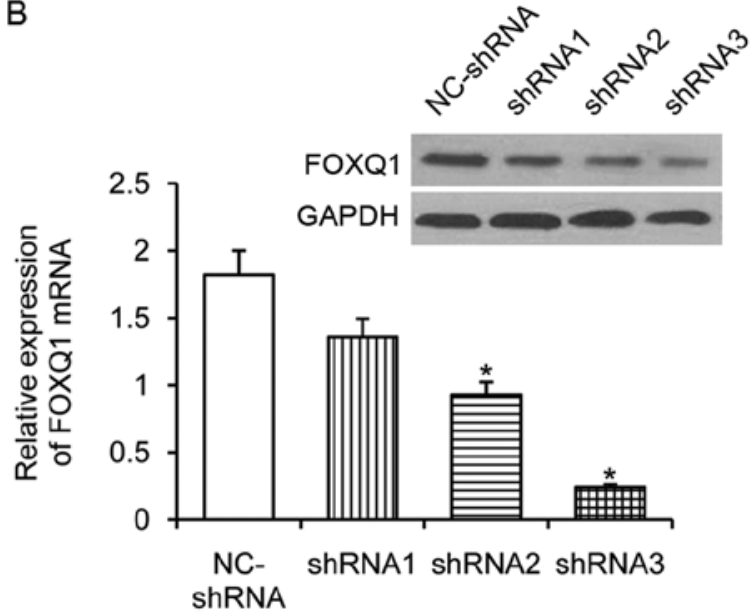

Figure 2. The expression of FOXQ1. (A) Quantitative RT-PCR and western blot analysis were performed to measure FOXQ1 mRNA and protein levels, respectively, in human bladder cancer cell lines T24 and BIU-87. High levels of FOXQ1 expression were observed in T24 cells compared to the BIU-87 cells. (B) Three positive plasmids (shRNA1, shRNA2 and shRNA3) targeted to FOXQ1 and one negative plasmid (NC-shRNA) were transfected into T24 cells at $1 \times 10^{6}$ per well which were cultured in 6-well plates. The relative levels of FOXQ1 mRNA transcripts in shRNA3 group to control GAPDH significantly decreased by qRT-PCR. Similar results could be obtained by western blot analysis for detecting FOXQ1. Data are expressed as mean \pm SD of each group of the cells. ${ }^{*} \mathrm{P}<0.05$ relative to the NC-shRNA group.

none-invasive epithelial bladder cancer BIU-87 cells. Similar results could be obtained by western blot analysis for detecting FOXQ1 (Fig. 2A). To unveil the possible role of FOXQ1 in the progression of bladder cancer, a more invasive cell line model T24 was chosen for knockdown expression of FOXQ1 by stable transfection of FOXQ1 shRNA. The three positive plasmids were named shRNA1, shRNA2, shRNA3; one negative plasmid was named NC-shRNA. To compare the effect of the shRNA-mediated silence of the target FOXQ1 gene expression, control shRNA, the FOXQ1-shRNAs and $50 \mathrm{nM}$ of each was transfected into T24 cells for $24 \mathrm{~h}$. The efficacy of individual inhibitory RNAs in downregulating the expression of FOXQ1 gene was measured by qRT-PCR. As shown in Fig. 2B, both the FOXQ1-shRNAs effectively inhibited the FOXQ1 gene transcription. However, the shRNA3 displayed the strongest inhibitory activity among these inhibitory shRNAs tested. The relative levels of FOXQ1 mRNA transcripts significantly decreased by nearly $90 \%$, as compared with the negative control group (NC-shRNA) and were significantly lower than those of other inhibitory RNAs $(\mathrm{P}<0.05)$. Expression of the internal standard GAPDH did not differ significantly between the groups $(\mathrm{P}>0.05)$. These data show that shRNA3 specifically inhibited the expression of FOXQ1 in T24 cells. Therefore, the FOXQ1-shRNA3 with the most effective silencing effect was chosen for knockdown expression of FOXQ1 and subsequent experiment.

FOXQ1 gene silencing induced changes in the morphology of T24 cells. Changes in transfection efficiency after $12-48 \mathrm{~h}$ were determined through the observation of green fluorescence; the strongest green fluorescence occurred at $48 \mathrm{~h}$. After transfection of recombinant plasmid through Lipofectamine 2000, the cells that had clear structure and form took on full green fluorescent protein in cytoplasm and nucleus (Fig. 3A). Interestingly, we found that NC-shRNA transfected T24 cells displayed irregular fibroblastoid morphology (Fig. 3B, left panel). In contrast, T24 cells treated with a reduced FOXQ1 expression appeared enlarged in cell size, while the integrity of the epithelial sheet structure remained intact. Compared to NC-shRNA group cells, FOXQ1-shRNA group T24 cells had a rounded shape, typical of an epithelial cobblestone appearance (Fig. 3B, right panel). These changes in phenotype suggested that the inhibition of FOXQ1 expression could potentially reverse EMT of T24 cells.

Changes in markers of epithelial and mesenchymal phenotypes. To further validate whether FOXQ1 gene silencing was able to reverse EMT phenotype in T24 cells, we determined the expression of epithelial marker E-cadherin (CDH1) and mesenchymal markers $\mathrm{N}$-cadherin and Vimentin using qRT-PCR and western blot analysis in FOXQ1-shRNA group and NC-shRNA group. Notably, FOXQ1 ablation induced a change from spindle-like mesenchymal morphology of T24 cells into epithelial morphology by manifesting an increased cell-to-cell adhesion (Fig. 2B). Consistent with the phenotypic change associated with FOXQ1 knockdown was an upregulation of epithelial marker E-cadherin concomitant with decreased expression of mesenchymal markers Vimentin and $\mathrm{N}$-cadherin, as determined at both mRNA and protein levels (Fig. 3C). Thus, FOXQ1 depletion resulted in a reversal of EMT in T24 cells, close to a normal epithelial phenotype and displayed a mesenchymal-epithelial transition (MET).

Effect of FOXQ1 gene silencing on the migration and invasion of T24 cells. FOXQ1 activity has been consistently correlated with the migratory and invasive potential of tumor cells (25). To functionally confirm the role of FOXQ1 in aggressive bladder cancers, we used a cell wound healing assay to examine the effect of FOXQ1 inhibition on cell migration in T24 cells. As shown in Fig. 4A, cells showed wounds that were healed nearly $48 \mathrm{~h}$ after scraping with pipette tips. Cells in the FOXQ1-shRNA group showed a reduced distance of migration compared with the NC-shRNA group. Furthermore, T24 cells freely invaded the matrigel and passed into the lower chamber in the NC-shRNA group. In contrast, treatment of the cells with FOXQ1-shRNA significantly reduced the number of cells that invaded (Fig. 4B), the difference was significant $(\mathrm{P}<0.05)$. Collectively, these findings indicated that the knockdown of FOXQ1 inhibited the migration and invasion of bladder cancer cells in vitro. 
A


B
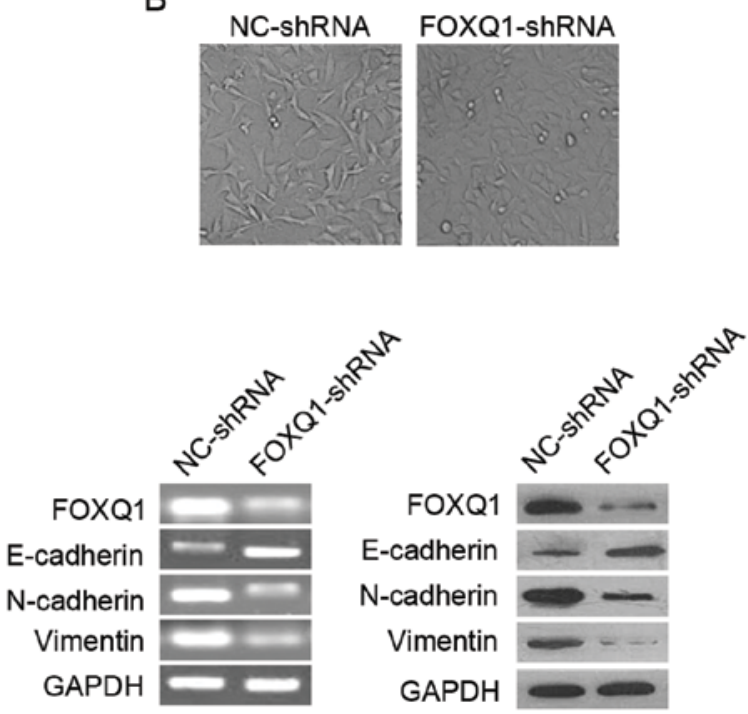

Figure 3. The knockdown of FOXQ1 induced a mesenchymal-epithelial transition-like phenotype in T24 cells. (A) The T24 cells that had clear structure successfully transfected with GFP-U6-shRNA plasmid and carried full green fluorescent protein in the cytoplasm and the nucleus. (B) Light microscopy visualization of T24 cells transfected with NC-shRNA or FOXQ1-shRNA. Original magnification, x200. Forty-eight hours after transfection, FOXQ1 knockdown cells appeared enlarged in size. Further, FOXQ1 knockdown cells maintained an epithelial sheet structure (right panel). (C) Knockdown expression of FOXQ1 differentially regulated the expression of EMT phenotype markers mRNA and protein and reversed the EMT phenotype.

A

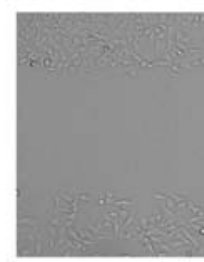

$\mathrm{Oh}$



NC-shRNA 48h FOXQ1-shRNA 48h

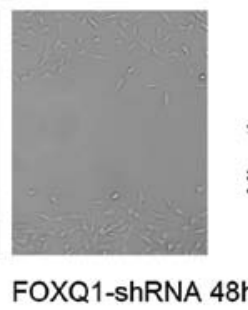



B
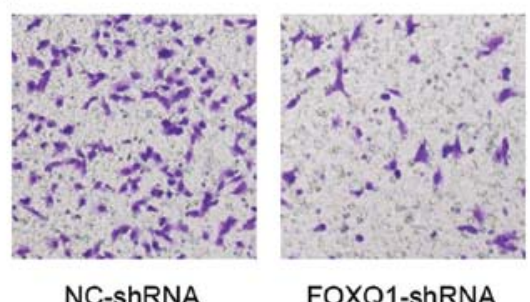

FOXQ1-shRNA

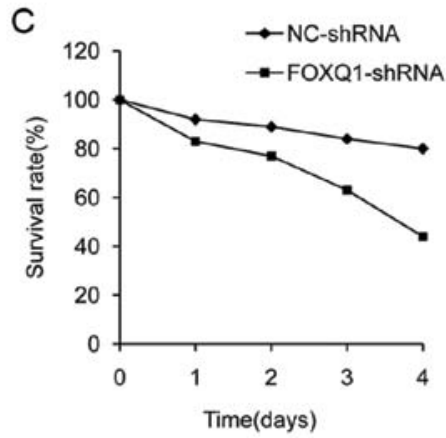

$\mathrm{D}$
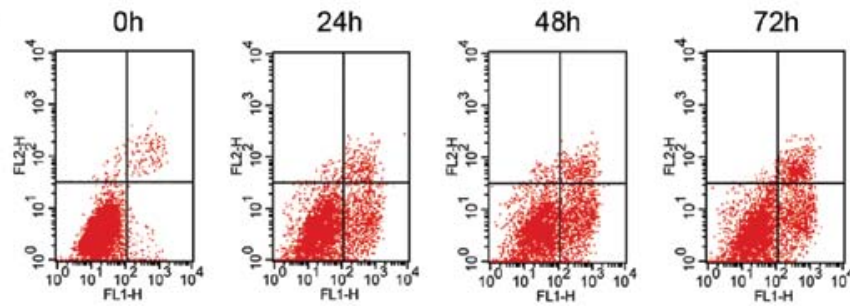

Figure 4. The cells were divided into two groups: FOXQ1-shRNA and NC-shRNA for cancer cell biological assays. (A) Wound healing assay was conducted to assess the capacity of cell migration and invasion in two group cells and the relative migration distance was shown in bar chart. ${ }^{*}<0.05$, relative to the NC-shRNA group. (B) Representative images of cells migrated to the bottom chamber showed that FOXQ1-shRNA group reduced the number of cells that invaded. (C) Cell viability of T24 cells was measured by MTT assay. The data are presented as means \pm standard deviation of 4 independent experiments and are expressed as a percentage of no-RNAi group. (D) Apoptosis analysis of T24 cells was performed by flow cytometry at 24,48 and $72 \mathrm{~h}$ after transfection. The apoptosis rates were $8.63 \pm 1.47,19.65 \pm 1.24$ and $26.82 \pm 1.35 \%$, respectively. 
The knockdown effect of FOXQ1 on cell viability and apoptosis. As shown in Fig. 4C, knockdown of FOXQ1 expression with the shRNA plasmid decreased cell viability of T24 cells by $24 \mathrm{~h}$ post-transfection, which was measured by MTT assay. FOXQ1 gene silencing promotes apoptosis in T24 cells. Flow cytometry analysis of T24 cells was performed 24, 48 and $72 \mathrm{~h}$ after transfection, the apoptosis rates were $(8.63 \pm 1.47$, $19.65 \pm 1.24$ and $26.82 \pm 1.35 \%$ ) respectively, and the cells at $72 \mathrm{~h}$ showed obvious apoptosis compared with cells of the 24-h group (Fig. 4D). These results suggest that our process of FOXQ1-shRNA plasmid transduction and FOXQ1 gene silencing in bladder cancer cells did suppress their viability.

\section{Discussion}

A great deal of literature indicates that EMT plays a pivotal role during embryonic development and in the formation of fibroblasts during inflammation and wound healing (26-28). Emerging evidence suggests that EMT also participates in the course of carcinogenesis. Accompanying with EMT process, cancer cells often acquire the capability to disseminate from the primary tumor site and to localise and group in distant organs, a process called metastasis (29). Recent studies have identified additional EMT regulators within the Forkhead transcription factor family and shown that of all 43 Forkhead family members presented in the array FOXQ1 was the most highly overexpressed in the highly invasive and mesenchymallike breast cancer MDA-MB-231 cells as compared to non-invasive epithelial-like breast cancer MCF7 cells (19). Consistently, we have shown that FOXQ1 was expressed in higher levels in aggressive bladder cancer T24 cells than that in non-aggressive bladder cancer BIU-87 cells.

In the present study, we have shown that the expression of TGF- $\beta 1$, FOXQ1 and Vimentin was significantly increased in human bladder cancer tissue compared to that of in background tissue. Patients with high TGF- $\beta 1$ (50/65) expression displayed strong FOXQ1 expression (58/65). Moreover, the patients with strong E-cadherin expression showed no or less staining of FOXQ1. A significant correlation between expression levels of FOXQ1 and E-cadherin was obvious in these human specimens $(\mathrm{P}<0.05)$. We have also shown that more patients with high Vimentin (54/65) expression displayed low E-cadherin expression (18/65), which confirmed a previous study (30). These results showed that there was an inverse relationship between FOXQ1 overexpression and loss of E-cadherin expression, a positive relationship between TGF- $\beta 1$ upregulated expression and FOXQ1 overexpression. FOXQ1 is one positive regulation factor for EMT phenotype, suggesting a potential impact of FOXQ1 expression regulated by TGF- $\beta 1$ signal pathway during EMT process of bladder tumor tissue.

Several reports have shown that EMT-inducing transcription factors such as SNAIL $(4,31)$, SIP1 (32), SLUG $(33,34)$, TWIST1 (35) repress expression of epithelial marker E-cadherin, and could act as important molecular regulators of EMT. Recent studies have identified that FOXQ1 repressed E-cadherin expression by targeting the E-box in its promoter region similarly to the E-cadherin repressors (17). E-cadherin plays a critical role in cell adhesion, development of epithelial organs and the maintenance of epithelial polarity (36). The progression of benign tumors to invasive metastatic cancer involves partial or complete loss of E-cadherin expression, or an impairment of its adhesive function (37). Direct transcriptional repression of E-cadherin was considered for transcription factors to trigger EMT (4), suggesting that FOXQ1, like other transcription factors, is also a critical mediator of EMT by repressing transcriptional activity of E-cadherin. FOXQ1 has become one of the few transcription factors involved in both EMT process and cancer metastasis.

To address the precise mechanism for FOXQ1 induced EMT-like phenotypic changes in bladder cancer cells, in this experiment, we designed independently and constructed shRNA directed to FOXQ1 and imported recombinant plasmid into bladder cancer T24 cells. The shRNA was dependent on the double-strand structure of the RNA and had target site specificity. We demonstrated initially that shRNA specific for FOXQ1 could be successfully transfected into T24 cells, resulting in significantly reduced gene transcription or expression level of protein. Interestingly, downregulation of FOXQ1 expression was accompanied by upregulation of E-cadherin and a lower level of $\mathrm{N}$-cadherin were observed in $\mathrm{T} 24$ cells treated with shRNA plasmid (Fig. 3C). This change in cadherin expression is referred to as 'cadherin-switch' (38). The observation that bladder cancer cells may gain mesenchymal characteristics, associated with the expression of mesenchymal markers (e.g., N-cadherin and Vimentin), has been proposed to contribute to the acquisition of the malignant phenotype $(39,40)$. In knockdown expression of FOXQ1, cancer cells expressing mesenchymal markers show cadherin-switch and reversal of EMT phenotype. However, the inhibition of Slug, Snail or Twist action through interfering RNA (siRNA or shRNA) resulted in tumor metastasis or growth inhibition (41-43), whether there is a possibility that FOXQ1 represses E-cadherin through regulating other EMT promoting genes is unclear, because mutual regulation is very common between the EMT promoting genes. These results strongly suggest that knockdown expression of transcription factors of EMT induction attain more effective multifunctional result. Therefore, transcription factors as therapeutic targets, compared with the inhibition of a single effector molecule or signal pathway is more reasonable and feasible.

In addition, the shRNA against FOXQ1 induced changes in the morphology of T24 cells, which were similar to a normal epithelial phenotype (Fig. 3B, right panel). Furthermore, knockdown of FOXQ1 expression caused a significant increase of multinuclear cells and significantly reduced the migration and invasion of bladder cancer cells in vitro assays (Fig. 4A and B), subsequently inhibiting proliferation and leading to cancer cell apoptosis (Fig. 4C and D). The result of our research suggested that RNA interference of FOXQ1 may inhibit metastasis of T24 cells through reversal of EMT.

In this study we found that knockdown expression of FOXQ1 leads to the reversal of EMT phenotype by upregulation in the protein expression of epithelial cell marker, E-cadherin and by downregulation of mesenchymal cell markers, $\mathrm{N}$-cadherin and Vimentin in T24 cells. These results strongly suggested the importance of FOXQ1 signaling in tumor cell aggressiveness through the acquisition of EMT phenotype in urothelium cancer cells. Therefore, targeting FOXQ1 signaling by novel approaches would be useful for reversing the EMT phenotype, which would likely result in the reversal of neoplasm recurrence and elimination of cancer cells. 


\section{Acknowledgements}

This study was supported by grants from the Chinese Natural Science Foundation (NSFC) (no. 30872561) to Zhaohui Zhu and (no. 30973008) to Yifei Xing.

\section{References}

1. Scheel C, Onder T, Karnoub A and Weinberg RA: Adaptation versus selection: the origins of metastatic behavior. Cancer Res 67: 11476-11480, 2007.

2. Thiery JP, Acloque H, Huang RY and Nieto MA: Epithelialmesenchymal transitions in development and disease. Cell 139 871-890, 2009

3. Thiery JP: Epithelial-mesenchymal transitions in development and pathologies. Curr Opin Cell Biol 15: 740-746, 2003.

4. Batlle E, Sancho E, Franci C, et al: The transcription factor snail is a repressor of E-cadherin gene expression in epithelial tumour cells. Nat Cell Biol 2: 84-89, 2000

5. Bolos V, Peinado H, Perez-Moreno MA, et al: The transcription factor slug represses E-cadherin expression and induces epithelial to mesenchymal transitions: a comparison with Snail and E47 repressors. J Cell Sci 116: 499-511, 2003.

6. Eger A, Aigner K, Sonderegger S, et al: DeltaEF1 is a transcriptional repressor of E-cadherin and regulates epithelial plasticity in breast cancer cells. Oncogene 24: 2375-2385, 2005.

7. Perez-Moreno MA, Locascio A, Rodrigo I, et al: A new role for E12/E47 in the repression of E-cadherin expression and epithelialmesenchymal transitions. J Biol Chem 276: 27424-27431, 2001.

8. Battula VL, Evans KW, Hollier BG, et al: Epithelial-mesenchymal transition-derived cells exhibit multilineage differentiation potential similar to mesenchymal stem cells. Stem Cells 28: $1435-1445,2010$

9. Myatt SS and Lam EW: The emerging roles of forkhead box (Fox) proteins in cancer. Nat Rev Cancer 7: 847-859, 2007.

10. Katoh M: Human FOX gene family. Int J Oncol 25: 1495-1500, 2004.

11. Hader C, Marlier A and Cantley L: Mesenchymal-epithelial transition in epithelial response to injury: the role of Foxc2. Oncogene 29: 1031-1040, 2010.

12. Bao B, Wang Z, Ali S, Kong D, Banerjee S, et al: Over-expression of FoxM1 leads to epithelial-mesenchymal transition and cancer stem cell phenotype in pancreatic cancer cells. J Cell Biochem 112: 2296-2306, 2011.

13. Bieller A, Pasche B, Frank S, Gläser B, Kunz J, et al: Isolation and characterization of the human forkhead gene FOXQ1. DNA Cell Biol 20: 555-561, 2001.

14. Feuerborn A, Srivastava PK, Küffer S, Grandy WA, Sijmonsma TP et al: The Forkhead factor FoxQ1 influences epithelial differentiation. J Cell Physiol 226: 710-719, 2011.

15. Cao D, Hustinx SR, Sui G, Bala P, Sato N, Martin S, et al: Identification of novel highly expressed genes in pancreatic ductal adenocarcinomas through a bioinformatics analysis of expressed sequence tags. Cancer Biol Ther 3: 1081-1091, 2004.

16. Paoni NF, Feldman MW, Gutierrez LS, Ploplis VA and Castellino FJ: Transcriptional profiling of the transition from normal intestinal epithelia to adenomas and carcinomas in the APCMin/t mouse. Physiol Genom 15: 228-235, 2003.

17. Kaneda H, Arao T, Tanaka K, Tamura D, Aomatsu K, et al: FOXQ1 is overexpressed in colorectal cancer and enhances tumorigenicity and tumor growth. Cancer Res 70: 2053-2063, 2010.

18. Qiao Y, Jiang X, Lee ST, Karuturi RK, Hooi SC, et al: FOXQ1 regulates epithelial-mesenchymal transition in human cancers. Cancer Res 71: 3076-3086, 2011.

19. Zhang H, Ethier SP, Miller F, Wu G, et al: Forkhead transcription factor Foxq1 promotes epithelial-mesenchymal transition and breast cancer metastasis. Cancer Res 71: 1292-1301, 2011.

20. Bubenick J, Baresora M, Viklicky V, et al: Established cell line of urinary bladder carcinoma (T24) containing tumor-specific antigen. Int J Cancer 11: 765-773, 1973.
21. Sobin DH and Witteking C: TNM Classification of Malignant Tumors. 6th edition. Wiley-Liss, New York, NY, 2003.

22. Miyamoto H, Miller JS, Fajardo DA, et al: Non-invasive papillary urothelial neoplasms: the $2004 \mathrm{WHO} / \mathrm{ISUP}$ classification system. Pathol Int 60: 1-8, 2010.

23. Yu Q, Zhang K, Wang X, Liu X and Zhang Z: Expression of transcription factors snail, slug, and twist in human bladder carcinoma. J Exp Clin Cancer Res 29: 119, 2010.

24. Shiozaki H, Tahara H, Oka H, Miyata M, et al: Expression of immunoreactive E-cadherin adhesion molecules in human cancers. Am J Pathol 139: 17-23, 1991.

25. Feng J, Zhang X, Zhu H, Wang X, Ni S and Huang J: FoxQ1 overexpression influences poor prognosis in non-small cell lung cancer, associates with the phenomenon of EMT. PLoS One 7: e39937, 2012

26. Peter ME: Let-7 and miR-200 microRNAs: guardians against pluripotency and cancer progression. Cell Cycle 8: 843-852, 2009.

27. Yang $\mathbf{J}$ and Weinberg RA: Epithelial-mesenchymal transition: at the crossroads of development and tumor metastasis. Dev Cell 14: 818-829, 2008.

28. Desmouliere A: Factors influencing myofibroblast differentiation during wound healing and fibrosis. Cell Biol Int 19: 471-476, 1995.

29. Hanahan D and Weinberg RA: Hallmarks of cancer: the next generation. Cell 144: 646-674, 2011.

30. Onder TT, Gupta PB, Mani SA, Yang J, Lander ES and Weinberg RA: Loss of E-cadherin promotes metastasis via multiple downstream transcriptional pathways. Cancer Res 68: 3645-3654, 2008.

31. Cano A, Perez-Moreno MA, Rodrigo I, et al: The transcription factor snail controls epithelial-mesenchymal transitions by repressing E-cadherin expression. Nat Cell Biol 2: 76-83, 2000.

32. Comijn J, Berx G, Vermassen P, et al: The two-handed E box binding zinc finger protein SIP1 downregulates E-cadherin and induces invasion. Mol Cell 7: 1267-1278, 2001.

33. Hajra KM, Chen DY and Fearon ER: The SLUG zinc-finger protein represses E-cadherin in breast cancer. Cancer Res 62: 1613-1618, 2002

34. De Craene B, van Roy F and Berx G: Unraveling signalling cascades for the Snail family of transcription factors. Cell Signal 17: 535-547, 2005.

35. Liu AN, Zhu ZH, Chang SJ, Hang XS, et al: Twist expression associated with the epithelial-mesenchymal transition in gastric cancer. Mol Cell Biochem 367: 195-203, 2012

36. Gumbiner BM: Cell adhesion: the molecular basis of tissue architecture and morphogenesis. Cell 84: 345-357, 1996.

37. Christofori $\mathrm{G}$ and Semb $\mathrm{H}$ : The role of the cell-adhesion molecule E-cadherin as a tumour-suppressor gene. Trends Biochem Sci 24: 73-76, 1999.

38. Zeisberg M and Neilson EG: Biomarkers for epithelial-mesenchymal transitions. J Clin Invest 119: 1429-1437, 2009.

39. McConkey DJ, Lee S, Choi W, et al: Molecular genetics of bladder cancer: emerging mechanisms of tumor initiation and progression. Urol Oncol 28: 429-440, 2010.

40. Iwatsuki M, Mimori K, Yokobori T, et al: Epithelial-mesenchymal transition in cancer development and its clinical significance. Cancer Sci 101: 293-299, 2010.

41. Zhang A, Chen G, Meng L, Wang Q, et al: Antisense-Snail transfer inhibits tumor metastasis by inducing E-cadherin expression. Anticancer Res 28: 621-628, 2008.

42. Cheng GZ, Chan J, Wang Q, et al: Twist transcriptionally upregulates AKT2 in breast cancer cells leading to increased migration, invasion, and resistance to paclitaxel. Cancer Res 67: 1979-1987, 2007

43. Vannini I, Bonafe M, Tesei A, et al: Short interfering RNA directed against the SLUG gene increases cell death induction in human melanoma cell lines exposed to cisplatin and fotemustine. Cell Oncol 29: 279-287, 2007. 